\title{
Der AKTX-Pflege e. V. stellt sich vor Plattform für Pflegekräfte

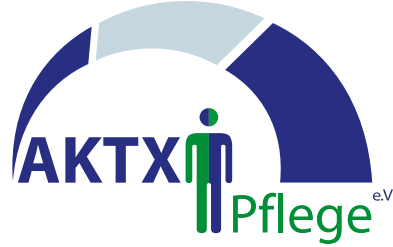 in der Transplantationsmedizin
}

Der AKTX-Pflege e. V. existiert seit Mitte 1999 und ist vorläufig als gemeinnütziger Verein anerkannt. Seither organisieren die Mitglieder Symposien zu pflegerelevanten Themen aus der Transplantationsmedizin und Patientenveranstaltungen. Außerdem veröffentlicht der Verein Beiträge in Pflegezeitschriften und dem Internet bzw. leistet bei der Kontaktaufnahme zu Pflegenden anderer Transplantationszentren Hilfestellung.

Im Folgenden gibt der AKTX-Pflege e. V. einen Überblick über seine Entstehung, Ziele und Tätigkeiten.

\section{Wer sind wir?}

Wir sind eine Gruppe von Pflegenden aus verschiedenen TX-Zentren (TX: Transplantation) Deutschlands. Angeregt durch internationale Organisationen haben wir uns zum Arbeitskreis Transplantationspflege zusammengeschlossen, um den Pflegekräften in Deutschland eine Plattform zu bieten, Probleme und Erfahrungen im Bereich der Transplantationspflege zu erörtern und Lösungsstrategien zu entwickeln.

Um den gestiegenen organisatorischen Ansprüchen gerecht zu werden, hat sich aus dem lockeren Zusammenschluss am 1. Juni 1999 der Verein AKTX-Pflege e.V. (AKTX: Arbeitskreis Transplantation) konstituiert. Der Verein wird vom Finanzamt vorläufig als gemeinnützig anerkannt.

\section{Was wollen wir?}

- qualifizierte Pflege in der Transplantationsmedizin

- Erfahrungs- und Informationsaustausch zwischen den Pflegenden der

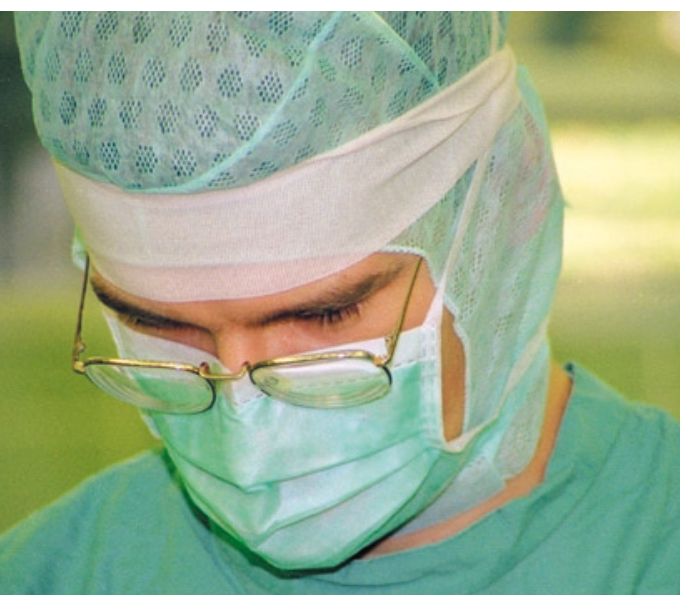

verschiedenen Transplantationszentren

- fachliche Fortbildungen zu Themen der Transplantationspflege

- Unterstützung von Pflegeforschungsprojekten in der Transplantationspflege

- Erarbeitung von Pflegekonzepten

- Zusammenarbeit mit internationalen und nationalen Fachverbänden, die im Sektor Transplantationspflege wirken, und den Patientenverbänden

\section{Was machen wir?}

- Wir veranstalten Symposien zu pflegerelevanten Themen aus der Transplantationsmedizin.

- Wir veröffentlichen Beiträge in Pflegezeitschriften und dem Internet.

- Wir geben Hilfestellung bei der Kontaktaufnahme zu Pflegenden anderer TX-Zentren.

- Wir organisieren Patientenveranstaltungen.

\section{Wie können Sie uns helfen?}

- Unterstützen Sie uns in den einzelnen Zentren, um den AKTX-Pflege in allen Transplantationsabteilungen bekannt zu machen.

- Bringen Sie Verbesserungs- und Themenvorschläge ein.

- Reichen Sie Beiträge für Pflegesymposien und Fachzeitschriften ein.

\section{Mitgliedsbeitrag}

Der Jahresmitgliedsbeitrag beläuft sich nach Beschluss der Mitgliederversammlung vom 22. 112001 auf 15 Euro.

\section{Wie sind wir zu erreichen?}

Im Internet: www.aktxpflege.de

Via E-Mail: info@aktxpflege.de 